\title{
Structural and electronic properties of isostructural transition metal nitrides
}

\author{
M. Sahnoun ${ }^{\mathrm{a}, *}$, J.C. Parlebas ${ }^{\mathrm{b}}$, M. Driz ${ }^{\mathrm{c}}$, C. Daul $^{\mathrm{d}}$ \\ ${ }^{a}$ Faculté des Sciences et Technologie, Université de Mascara, DZ-29000 Mascara, Algeria \\ b IPCMS, UMR 7504 CNRS-ULP, 23 Rue de Loess, 67034 Strasbourg Cedex 2, France

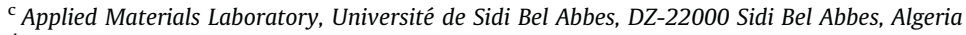 \\ d Département de Chimie, Université de Fribourg, CH-1700 Fribourg, Switzerland
}

\begin{abstract}
The structural and electronic properties of three isostructural transition metal nitrides $\mathrm{VN}, \mathrm{NbN}$ and TaN have been calculated using the full-potential linearized augmented plane-wave method within a generalized gradient approximation scheme for the exchange-correlation potential. Perfect $\mathrm{NaCl}$ structures as well as lattices containing nitrogen vacancies $\left(\mathrm{M}_{4} \mathrm{~N}_{3}\right)$, and an hexagonal $\varepsilon-\mathrm{M}_{2} \mathrm{~N}$ structure are considered. The trends are discussed and compared with available experimental and other theoretical results.
\end{abstract}

\section{Introduction}

Transition metal nitrides (TMNs) are hard refractory materials that are used in multiple types of applications [1-7] because of their renowned hardness [4,5], excellent thermal stability $[1,2,8,9]$, defect impermeability [6] and resistance to wear, oxidation and corrosion. These compounds normally exist in substoichiometric phases with a substantial amount of vacancies [10-12]. Non-metal deficiency is known to be a major source of the non-stoichiometry, and the control of the stoichiometry and the understanding of its effect on mechanical properties are crucial to the design of hard materials based on these compounds. Moreover, the electronic structures should be sensitive to the presence of vacancies and differ significantly from those for the stoichiometric phase. The vacancy concentration as well as the alloy composition is shown crucial to the electronic and mechanical properties in these materials $[13,14]$. However, there are still some unresolved questions to be discussed. In our opinion, the vacancy effects in these compounds need more profound investigations; detailed calculations are required to understand the mechanical and electronic properties of the substoichiometric transition metal compounds. Among these compounds, there is increasing interest on vanadium nitride as an important industrial catalyst known for its selectivity and stability [15,16]. Also VN is a superconductor with transition

\footnotetext{
* Corresponding author.

E-mail address: sahnoun_cum@yahoo.fr (M. Sahnoun).
}

temperature ranging from 2 to $9 \mathrm{~K}$ [17]. In the phase diagram of $\mathrm{V}-\mathrm{N}$, several binary phases have been reported: vanadium mononitride VN, which exhibits the rock salt structure and exists over the compositions from $\mathrm{VN}_{0.7}$ to $\mathrm{VN}_{1.0}$ [18]. In a previous paper [19], X-ray diffraction (XRD) measurements of VN samples showed that films deposited at $150{ }^{\circ} \mathrm{C}$ and at low nitrogen gas flows exhibit a hexagonal sub-nitride phase $\varepsilon-\mathrm{V}_{2} \mathrm{~N}$. Hofer et al. [20] have also reported a rapid thermal processing of vanadium layers in pure nitrogen at high temperature, and their electron diffraction patterns revealed different phases: a rock salt structure was identified at $1100{ }^{\circ} \mathrm{C}$ and vanadium sub-nitride with the hexagonal structure $\left(\varepsilon-\mathrm{V}_{2} \mathrm{~N}\right)$ was found at $900{ }^{\circ} \mathrm{C}$. This latter phase was firstly identified by Christensen and Lebech [21]. Similarly, the phase diagram for niobium-nitrogen system is also complex and only partly investigated, in which three phases are stable up to $2100{ }^{\circ} \mathrm{C}$ : a trigonal $\beta-\mathrm{Nb}_{2} \mathrm{~N}$, a tetragonal $\gamma-\mathrm{NbN}$, and the cubic rock salt $\delta$-TaN. All three compounds are non-stoichiometric $[22,23]$. $\delta$-NbN is metallic and becomes superconductive at $T_{c}=17.3 \mathrm{~K}$ [24]. Moreover, the Ta-N equilibrium phase diagram is extremely rich [25-28] and relatively unexplored, in which four phases are stable up to $1860{ }^{\circ} \mathrm{C}$ : $\varepsilon-\mathrm{TaN}, \delta-\mathrm{TaN}_{1-x}, \beta-\mathrm{Ta}_{2} \mathrm{~N}_{1-x}$ and $\alpha-\mathrm{Ta}(\mathrm{N})$ [29]. The cubic rock salt structure $\left(\delta-\mathrm{TaN}_{1-x}\right)$ is obtained over a wide range of compositions from $\mathrm{TaN}_{0.69}$ to $\mathrm{TaN}_{1.04}$. Theoretically, Papaconstantopoulos et al. [30] also predicted that cubic TaN has high superconducting critical temperature.

These materials exist over a wide range of compositions outside stoichiometry and their physical properties vary as a consequence. Despite many physical properties, previously determined for cubic $\mathrm{MN}(\mathrm{M}=\mathrm{V}, \mathrm{Nb}$ and $\mathrm{Ta})$ and summarized in 
Ref. [31], no studies have been yet determined for the stable phase $\beta-M_{2} N(M=V, N b$ and $T a)$. These substoichiometric compounds exhibit a hexagonal $\varepsilon$ - $\mathrm{Fe}_{2} \mathrm{~N}$-type structure with a space group $\mathrm{P}-31 \mathrm{~m}$ [32]. The aim of this paper is to present the results of a theoretical investigation of the structural and electronic characteristics of a bulk of three isostructural compounds $\mathrm{VN}, \mathrm{NbN}$ and TaN, which crystallize in a cubic $\mathrm{NaCl}$-type and in a hexagonal $\varepsilon-\mathrm{Fe}_{2} \mathrm{~N}$-type lattices. The $\varepsilon-\mathrm{Fe}_{2} \mathrm{~N}$ structure is hexagonal; the space group is P-31m (no. 162) with three formula units per unit cell. The metal atoms can be placed in the $6 \mathrm{k}$ site with $(0.323,0.0$, 0.272 ) coordinates, and the nitrogen atoms can be placed in the $2 \mathrm{~d}$ and in the $1 \mathrm{a}$ sites with $(1 / 3,2 / 3,1 / 2)$ and $(0.0,0.0,0.0)$ coordinates, respectively. In addition to these two phases $(\delta$-MN and $\beta-\mathrm{M}_{2} \mathrm{~N}$ ), which have been observed in nature, we performed calculations for another substoichiometric rock salt structure $\left(\mathrm{M}_{4} \mathrm{~N}_{3}\right)$ for comparison purpose.

The paper is organized as follows: we explain the computational method in Section 2. The results are presented and discussed in Section 3 for the electronic structure properties. A brief conclusion is drawn in Section 4 .

\section{Computational method}

The electronic structure calculations were performed using the full linearized augmented plane-wave method using the package Wien $2 k$ [33]. The unit cell is separated into non-overlapping atomic spheres and an interstitial region. The wave functions are described using radial solutions of Schrödinger's equation inside the spheres and plane waves in the interstitial regions. Exchange and correlation effects were treated using the generalized gradient approximation (GGA) [34]. For each calculation, typically, more than 100k-points were used in the irreducible
Brillouin zone, a maximum angular momentum for the radial wave functions ( $\left.l_{\max }\right)$ of 12 and a plane-wave cutoff $\left(R_{\mathrm{MT}} K_{\max }\right)$ of 8 . Local orbitals were added to the basis set. This allows a better treatment of the problem of semicore states present in transition metal compounds; semicore states are high lying, relatively extended core states (e.g., the M p states) [35]. Also, addition of extra local orbitals, e.g., non-metal s and $\mathrm{p}$ and metal d orbitals, at energies high above the Fermi energy, enables a better and more reliable description of the unoccupied states. We have chosen the muffin-tin radii $\left(R_{\mathrm{MT}}\right)$ for $\mathrm{V}, \mathrm{Nb}$ and Ta to be 2.09 (1.93), 2.05 (1.93) and 2.01 (1.91) a.u., respectively, in the cubic (hexagonal) structures of $\mathrm{VN}\left(\mathrm{V}_{2} \mathrm{~N}\right), \mathrm{NbN}\left(\mathrm{Nb}_{2} \mathrm{~N}\right)$ and $\mathrm{TaN}\left(\mathrm{Ta}_{2} \mathrm{~N}\right)$, and finally 1.85 (1.85) a.u. for the nitrogen atom. We compute lattice constants and bulk moduli by fitting the total energy versus volume according to the Murnaghan's equation of state [36]. The total density of states (DOS) was obtained using a modified tetrahedron method of Blöchl et al. [37]. A non-stoichiometric $\mathrm{MN}_{0.75}$ was also simulated by substituting a vacancy on one nonmetal $\mathrm{N}$ atom in unit cell of 8 atoms in a $\mathrm{NaCl}$ structure.

\section{Results and discussions}

We have calculated the ground state properties for transition metal (TM) nitrides. Results are summarized in Table 1 and compared with available experiments and results of other calculations. We have found excellent agreement between our calculated and available experiment and theoretical lattice parameters for all the compounds. Our calculated bulk moduli are in very good agreement with most of the available data. From Table 1 the agreement between our results and the pseudopotential calculations of Isaev et al. [31] appears to be excellent for both lattice parameter and bulk moduli.

Table 1

Ground state parameters for transition metal nitrides within cubic and hexagonal structures. Results of other calculations and experimental data are shown on the next line after results of our calculations.

\begin{tabular}{|c|c|c|c|c|}
\hline Compounds & $\mathbf{a}(\AA)$ & c (Å) & $V\left(\AA^{3}\right)$ & B (GPa) \\
\hline \multirow[t]{2}{*}{$\delta$-VN } & 4.12 & & 69.934 & 305 \\
\hline & $4.11^{\mathrm{a}}, 4.136^{\mathrm{b}}, 4.12^{\mathrm{c}}, 4.14^{\mathrm{d}, \mathrm{e}}, 4.132^{\mathrm{f}}, 4.19^{\mathrm{g}}, 4.092^{\mathrm{h}}$ & & & $313^{\mathrm{a}}, 333^{\mathrm{c}}, 316^{\mathrm{f}}, 282^{\mathrm{e}}, 338^{\mathrm{g}}, 370^{\mathrm{h}}, 268^{\mathrm{i}}$ \\
\hline $\mathbf{V}_{4} \mathbf{N}_{3}$ & 4.07 & & 67.419 & 285 \\
\hline \multirow[t]{2}{*}{$\beta-V_{2} N$} & 4.98 & 4.53 & 97.294 & 258 \\
\hline & $4.917^{\mathrm{j}}$ & $4.568^{\mathrm{j}}$ & & \\
\hline \multirow[t]{2}{*}{$\delta-\mathrm{NbN}$} & 4.43 & & 87.275 & 314 \\
\hline & $4.41^{\mathrm{a}}, 4.394^{\mathrm{b}}, 4.42^{\mathrm{c}}, 4.378^{\mathrm{g}}, 4.392^{\mathrm{k}}, 4.379^{1}$ & & & $309^{\mathrm{a}}, 317^{\mathrm{c}}, 350^{\mathrm{m}}, 292^{\mathrm{n}}, 287^{\mathrm{i}}, 354^{\mathrm{n}, \mathrm{o}}$ \\
\hline $\mathrm{Nb}_{4} \mathrm{~N}_{3}$ & 4.39 & & 84.604 & 282 \\
\hline \multirow[t]{2}{*}{$\beta-\mathrm{Nb}_{2} \mathrm{~N}$} & 5.39 & 4.99 & 125.547 & 263 \\
\hline & $5.267^{p}$ & $4.988^{\mathrm{p}}$ & & \\
\hline \multirow[t]{2}{*}{$\delta$-TaN } & 4.43 & & 87.275 & 332 \\
\hline & $4.408^{\mathrm{a}}, 4.33^{\mathrm{b}}, 4.42^{\mathrm{c}}, 4.397^{\mathrm{l}}, 4.385^{\mathrm{c}}, 4.336^{\mathrm{q}}$ & & & $329^{\mathrm{a}}, 338^{\mathrm{c}}, 372^{\circ}$ \\
\hline $\mathrm{Ta}_{4} \mathrm{~N}_{3}$ & 4.38 & & 84.027 & 304 \\
\hline$\beta-\mathbf{T a}_{2} \mathbf{N}$ & 5.38 & 4.98 & 124.831 & 265 \\
\hline
\end{tabular}

${ }^{a}$ Ref. [31] (Calc.)

${ }^{b}$ Ref. [38] (Exp.)

${ }^{c}$ Ref. [39] (Calc.)

${ }^{d}$ Ref. [40] (Exp.)

e Ref. [41] (Calc.)

${ }^{f}$ Ref. [42] (Calc.)

${ }^{g}$ Ref. [43] (Calc.)

${ }^{\text {h }}$ Ref. [44] (Calc.)

${ }^{\mathrm{i}}$ Ref. [45] (Exp.)

j Ref. [21] (Calc.)

${ }^{\mathrm{k}}$ Ref. [46] (Calc.)

${ }^{1}$ Ref. [20] (Exp.)

${ }^{m}$ Ref. [47] (Exp.)

${ }^{n}$ Ref. [48] (Calc.)

${ }^{\circ}$ Ref. [49] (Calc.)

p Ref. [22] (Calc.)

${ }^{\mathrm{q}}$ Ref. [50] (Exp.) 
There are some general trends in lattice parameters and bulk modulus we would like to point out. The first trend is that the bulk moduli of the metal nitrides are very close to each other. The second trend is a decrease in the bulk moduli with nitrogen vacancy (from $\mathrm{MN}$ to $\mathrm{M}_{4} \mathrm{~N}_{3}$ ), and obviously $\mathrm{N}$ vacancies make these compounds more compressible. Furthermore, we observed a change in the lattice parameters depending on the composition of the non-stoichiometric compound $\mathrm{MN}_{1-x}(\mathrm{M}=\mathrm{V}, \mathrm{Nb}$ and $\mathrm{Ta})$; in the rock salt-type structure, each metal atom has an octahedral environment of six sites in the nitrogen sublattice, while each site in the nitrogen sublattice is surrounded by six metal atoms. The presence of one or several structural vacancies in the nearest environment of a metal atom causes static displacements of this atom due to combined asymmetry effect of its nearest neighbors. Meanwhile the metal-nitrogen interactions in adjacent occupied octahedra will prevent the metal atoms to move continually towards a vacancy, which will be accompanied by a decrease in the equilibrium lattice parameter. Consequently, new chemical bonds are responsible for the changes in the cohesion of the considered non-stoichiometric compounds. Thus, if bulk moduli decrease as a function of the vacancy concentration, it is a consequence of the formation of metal-metal bonds.

We have investigated the electronic band structures along the various symmetry lines and also the density of states (DOS) of stoichiometric and substoichiometric transition metal nitrides within cubic and hexagonal structures, in order to understand differences in the chemical bonding between them. In the case of stoichiometric MN (Fig. 1), the overall band profiles are found to be the same for the three compounds and are in good agreement with other band structures calculations $[51,52]$. The density of states (DOS) of the lowest bands, with energy in the range from -15 to $-18 \mathrm{eV}$ in the three compounds, is mainly composed of $\mathrm{N}$ $2 \mathrm{~s}$ states. There is a gap of about $5 \mathrm{eV}$ between the $\mathrm{N} 2 \mathrm{~s}$ states and the bottom of the valence band. The valence band is mainly composed of $\mathrm{N} 2 \mathrm{p}$ and the metal $\mathrm{d}$ states, which coincide in the energy positions and the shape of curves indicating a strong covalent interaction between the metal and the nitrogen for the three compounds. Finally, at higher energies, above the Fermi level, there are the metal d bands, which are mostly mixed with nitrogen $2 p$ states. When vacancies are included, in the case of hexagonal $\beta-\mathrm{M}_{2} \mathrm{~N}$ and cubic $\mathrm{M}_{4} \mathrm{~N}_{3}$ (Fig. 1), the curves of DOS show an intensity decrease near $-7 \mathrm{eV}$ that can be understood as a reduction in $\mathrm{N} 2 \mathrm{p}$ derived states in this region. Also, we note a shift of the valence band toward $E_{F}$ with nitrogen vacancy, which is interpreted with the idea that a decrease in nitrogen atoms results in additional negative charge in the vicinity of the metal atoms. Moreover, additional structures appear: additional peaks are observed at a range of $2.5 \mathrm{eV}$ below the Fermi level. These peaks can be interpreted as being originated from vacancy-induced states. These vacancy peaks are attributed to modifications of the metal density of states. Their origin can be explained by symmetry changes resulting from the vacancy sites in the lattice. The metal orbitals point to the nearest neighbor on the nitrogen sublattice and are particularly affected by the introduction of vacancies. Even a
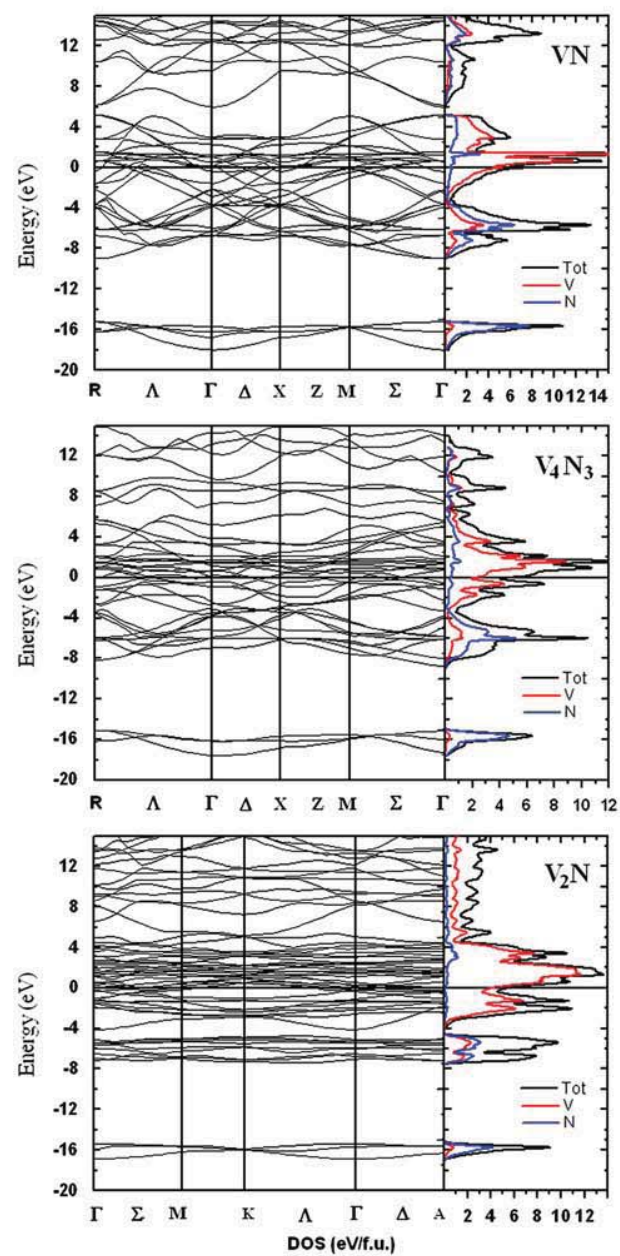

b
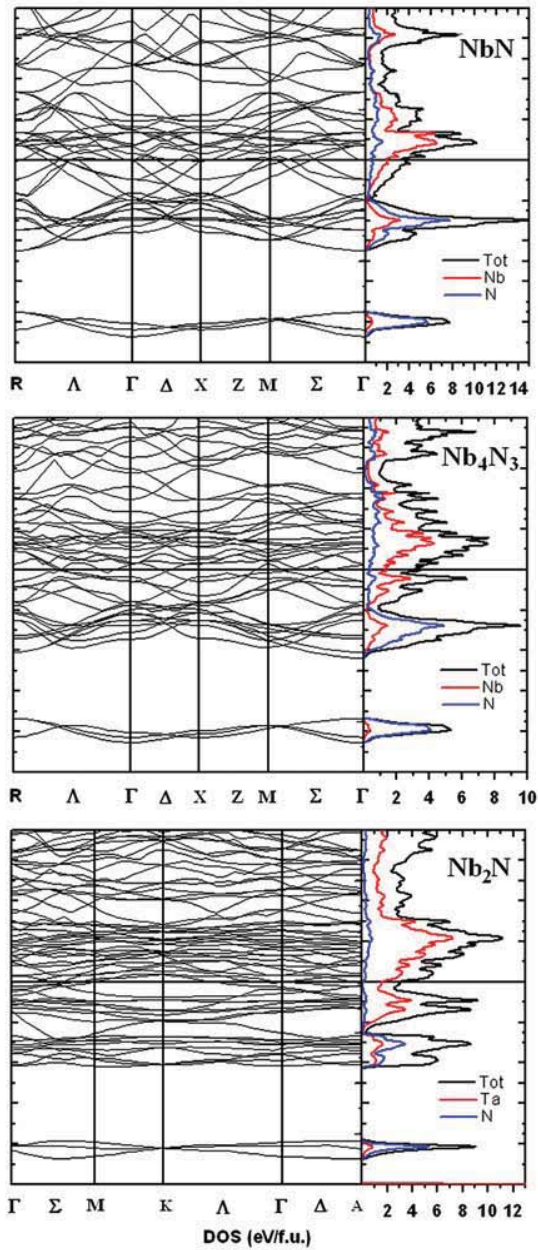

C
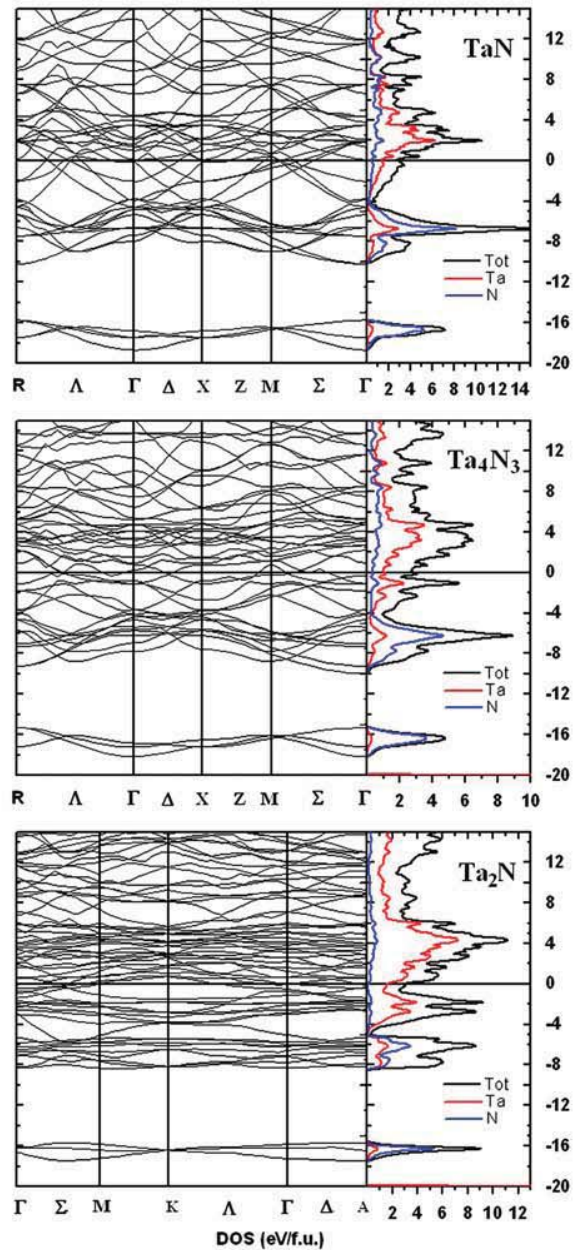

Fig. 1. FP-LAPW band structure and densities of states of $\delta-M N, M 4 N 3$, and $\beta-M_{2} N(M=V, N b$ and Ta). The energy zero is taken at Fermi level. 
then the peaks above the Fermi level with mainly metal $d$ character are also affected by the introduction of vacancies. Moreover, this interpretation is in good agreement with Skala and Capkova [53] on substoichiometric vanadium nitrides where there are also similar important $\mathrm{V}-\mathrm{V}$ bonds through vacancies.

\section{Conclusion}

We have used the new FP-LAPW method, within the GGA for the exchange-correlation potential, to investigate the electronic properties distinguishing between three structurally and chemically different transition metal nitrides $\mathrm{MN}$, namely $\delta-\mathrm{MN}, \mathrm{M}_{4} \mathrm{~N}_{3}$ and $\beta-M_{2} N(M=V, N b$ and $T a)$. The geometry of these three structures has been optimized and good agreement is obtained with experiment and other calculation data. For the three transition metal nitrides, $\mathrm{VN}, \mathrm{NbN}$ and $\mathrm{TaN}$, deviations from stoichiometry strongly affect the energy distribution curve of the valence band. There is a significant redistribution of the metal $d$ states near the Fermi level. The appearance of vacancy structures indicates that new bonds are formed between the metal atoms surrounding the nitrogen vacancy. Our calculations also indicate that the vacancy states are common for the entire family of considered transition metal nitrides ( $\mathrm{VN}, \mathrm{NbN}$ and $\mathrm{TaN}$ ), but the energy and width of such states depend strongly on the particular compound.

\section{References}

[1] L. Hultman, Vacuum 57 (2000) 1

[2] I. Pollini, A. Mosser, J.C. Parlebas, Phys. Rep. 355 (2001) 1.

[3] H. Hochst, R.D. Bringans, P. Steiner, T. Wolf, Phys. Rev. B 25 (1982) 7183.

[4] A.J. Perry, J. Vac. Sci. Technol. A 6 (1988) 2140.

[5] S.H. Jhi, J. Ihm, S.G. Louie, M.L. Cohen, Nature (London) 399 (1999) 132.

[6] D.J. Kim, Y.B. Jung, M.B. Lee, Y.H. Lee, J.H. Lee, J.H. Lee, Thin Solid Films 372 (2000) 276.

[7] C.S. Shin, D. Gall, N. Hellgren, J. Patscheider, I. Petrov, J.E. Greene, J. Appl. Phys. 93 (2003) 6025

[8] L. Tsetseris, N. Kalfagiannis, S. Logothetidis, S.T. Pantelides, Phys. Rev. Lett. 99 (2007) 125503.

[9] L. Tsetseris, N. Kalfagiannis, S. Logothetidis, S.T. Pantelides, Phys. Rev. B 76 (2007) 224107.

[10] L.E. Toth, in: Transition Metal Carbides and Nitrides, Academic Press, New York, 1971.

[11] H. Holleck, J. Vac. Sci. Technol. A 4 (1986) 2661

[12] W.S. Williams, Mater. Sci. Eng. A 105/106 (1988) 1.

[13] R. Sanjins, C. Wiemer, P. Hones, F. Lvy, J. Appl. Phys. 83 (1998) 1396

[14] M. Guemmaz, G. Moraitis, A. Mosser, M.A. Khan, J.C. Parlebas, J. Phys. Condens. Matter 9 (1997) 8453.

[15] H. Kwon, S. Choi, L.T. Thompson, J. Catal. 184 (1999) 236.
[16] S.T. Oyama, Catal. Today 15 (1992) 179.

[17] B. Massalski, in: Binary Alloy Phase Diagram, ASM, Metals Park, OH, 1991.

[18] M. MacKenzie, A.J. Parkin, A. Nartowski, in: ELNES in Metal Nitrides. Conference Series 153, Institute of Physics Publishing Ltd., Bristol, 1997 323.

[19] H. Gueddaoui, G. Schmerber, M. Abes, M. Guemmaz, J.C. Parlebas, Catal. Today 113 (2006) 270.

[20] E. Hofer, P. Warbichler, A. Scott, R. Brydson, I. Galesic, B. Kolbesen, J. Microsc. 204 (2001) 166

[21] A.N. Christensen, B. Lebech, Acta Cryst. B 35 (1979) 2677.

[22] A.N. Christensen, Acta Chim. Scand. A 30 (1976) 219.

[23] K. Yamada, H. Masuda, N. Sato, T. Fujino, J. Solid State Chem. 150 (2000) 36.

[24] G. Brauer, H. Kirner, Z. Anorg. Allg. Chem. 328 (1964) 34

[25] G.R. Lee, J.J. Lee, C.S. Shin, I. Petrov, J.E. Greene, Thin Solid Films 475 (2005) 45

[26] T.B. Massalski, in: Binary Alloys Phase Diagrams, ASM International, Ohio, 1990, pp. 2703.

[27] N. Terao, Jpn. J. Appl. Phys. 10 (1971) 248.

[28] D. Gerstenberg, C.J. Calbick, J. Appl. Phys. 35 (1964) 402.

[29] H. Wiesenberger, W. Lengauer, P. Ettmayer, Acta Mater. 46 (1998) 659.

[30] D.A. Papaconstantopoulos, W.E. Pickett, B.M. Klein, L.L. Boyer, Phys. Rev. B 31 (1985) 752.

[31] E.I. Isaev, S.I. Simak, A. Abrikosov, R. Ahuja, Yu. Kh., M.I. Vakilov A.I. Katsnelson, Lichtenstein, B. Johansson, J. Appl. Phys. 101 (2007) 123519

[32] I.E. Toth, in: Transition Metal Carbides and Nitrides, Academic Press, New York, 1971.

[33] P. Blaha, K. Schwarz, G.K.H. Madsen, D. Kvasnicka, J. Luitz, in: WIEN2k, An Augmented Plane Wave Plus Local Orbitals Program for Calculating Crystal Properties, Vienna University of Technology, Vienna, Austria, 2001.

[34] J.P. Perdew, S. Burke, M. Ernzerhof, Phys. Rev. Lett. 76 (1996) 3865

[35] D. Singh, Plane Waves, in: Pseudopotentials and the LAPW Method, Kluwer, Dordrecht, 1994

[36] F.D. Murnaghan, Proc. Natl. Acad. Sci. USA 30 (1944) 5390.

[37] P. Blöchl, O. Jepsen, O.K. Anderson, Phys. Rev. B 49 (1994) 16223

[38] P. Villars and L.D. Calvet, Pearson's Handbook of Crystallographic Data for Intermetallic Phases (American Society for Metals, Metals Park, OH, 1985).

[39] C. Stampfl, W. Mannstadt, R. Asahi, A.J. Freeman, Phys. Rev. B 63 (2001) 155106.

[40] P.S. Herle, M.S. Hegde, N.Y. Vasathacharya, S. Philip, M.V. Rama Rao T. Sripathi, J. Solid State Chem. 134 (1997) 120.

[41] V.P. Zhukov, V.A. Gubanov, O. Jepsen, N.E. Christensen, O.K. Anderson, J. Phys. Chem. Solids 49 (1988) 841.

[42] D.J. Singel, L.G. Hector, J.B. Adams, Phys. Rev. B 67 (2003) 092105.

[43] J.C. Grossman, A. Mizel, M. Côté, M.L. Cohen, S.G. Louie, Phys. Rev. B 60 (1999) 6343.

[44] W. Wolf, R. Poudloucky, T. Antretter, F.D. Fisher, Philos. Mag. B 79 (1999) 839.

[45] J.O. Kim, J.D. Achenbach, P.B. Mirkarimi, N. Shinn, S.A. Barnett, J. Appl. Phys. $72(1992) 1805$.

[46] A.F. Guillermet, J. Hägland, G. Grimvall, Phys. Rev. B 48 (1993) 1167345 (1992) 11557.

[47] G.L.W. Hart, B.M. Klein, Phys. Rev. B 61 (2000) 3151.

[48] X.-I. Chen, et al., Proc. Natl. Acad. Sci. USA 102 (2005) 3198.

[49] Z. Wu, X.-I. Chen, V.V. Struzhkin, R.E. Cohen, Phys. Rev. B 71 (2005) 214103

[50] T. Mashimo, S. Tashiro, T. Toya, M. Nishida, H. Yamazaki, S. Yamara, K. Oh-Ishi Y. Syono, J. Mater. Sci. 28 (1993) 3349.

[51] H.B. Nie, S.Y. Xu, S.J. Wang, L.P. You, Z. Yang, C.K. Ong, J. Li, T.Y.F. Liew, Appl. Phys. A 73 (2001) 229

[52] M. Sahnoun, C. Daul, M. Driz, J.C. Parlebas, C. Demangeat, Comput. Mat. Sci. 33 (2005) 175.

[53] L. Skala, P. Capkova, J. Phys.: Condens. Matter 2 (1990) 8293. 